\title{
Supernormal conduction in cardiac tissue promotes concordant alternans and action potential bunching
}

\author{
Blas Echebarria, ${ }^{1}$ Georg Röder, ${ }^{2}$ Harald Engel, ${ }^{3}$ Jörn Davidsen, ${ }^{4}$ and Markus Bär ${ }^{5}$ \\ ${ }^{1}$ Departament de Física Aplicada, Universitat Politècnica de Catalunya, E-08028 Barcelona, Spain \\ ${ }^{2}$ Max-Planck-Institut für Physik komplexer Systeme, D-01187 Dresden, Germany \\ ${ }^{3}$ Institut für Theoretische Physik, Technische Universität Berlin, D-10623 Berlin, Germany \\ ${ }^{4}$ Department of Physics and Astronomy, University of Calgary, Calgary, Alberta T2N 1N4, Canada \\ ${ }^{5}$ Physikalisch-Technische Bundesanstalt, D-10587 Berlin, Germany \\ (Received 23 July 2010; revised manuscript received 22 November 2010; published 4 April 2011)
}

\begin{abstract}
Supernormal conduction (SNC) in excitable cardiac tissue refers to an increase of pulse (or action potential) velocity with decreasing distance to the preceding pulse. Here we employ a simple ionic model to study the effect of SNC on the propagation of action potentials (APs) and the phenomenology of alternans in excitable cardiac tissue. We use bifurcation analysis and simulations to study attraction between propagating APs caused by SNC that leads to AP pairs and bunching. It is shown that SNC stabilizes concordant alternans in arbitrarily long paced one-dimensional cables. As a consequence, spiral waves in two-dimensional tissue simulations exhibit straight nodal lines for SNC in contrast to spiraling ones in the case of normal conduction.
\end{abstract}

DOI: 10.1103/PhysRevE.83.040902

PACS number(s): 87.19.Hh, 05.45.-a, 87.19.R-

Propagation of pulse trains in excitable media is usually characterized by a so-called dispersion curve that gives the velocity of a pulse in a periodic train as a function of its wavelength [1]. In cardiac tissue the dispersion properties are often summarized in the conduction velocity $(\mathrm{CV})$ restitution curve that relates the velocity of an action potential (AP) to its diastolic interval (DI), that is, the time between two consecutive APs (see, e.g., Ref. [2]).

Since a pulse typically is followed by a refractory zone of decreased excitability, the pulse velocity monotonically increases with increasing wavelength (=normal dispersion). In the context of excitable cardiac tissue, normal dispersion corresponds to normal conduction. In this paper we focus on the alternative situation where the velocity of a pulse is decreasing with increasing wavelength. In generic excitable media this case is known as anomalous dispersion. For excitable cardiac tissue it corresponds to supernormal conduction (SNC) [3]. Normal and supernormal conduction may appear for a different wavelength in the same systems, then the dispersion curve has to be nonmonotonic.

The simplest case of a nonmonotonic dispersion relation is a curve with a single maximum separating anomalous dispersion at long wavelength from normal dispersion at short wavelength. Such behavior has been discovered in various experiments in chemical reaction-diffusion systems [4,5]. It has been found to coincide with attractive long-range interaction between pulses that may lead to stable bound states [6] merging or bunching of pulses [7]. While most excitable cardiac tissues and related models exhibit normal conduction, nevertheless examples of SNC are frequent (for a short review see, e.g., Ref. [8]). SNC is found, for example, in the Iyer-Mazhari-Winslow [9] or in the Drouhard-RobergeBeeler-Reuter (DRBR) model [10,11] employed in the study here, and it is often observed under conditions of low extracellular potassium concentration [12]. Recently, it has been shown to potentiate the onset of alternans in cultured cardiac cell strands [13] and also in generic models of spiral wave formation [14].
Alternans is an oscillation in the duration of the AP that usually occurs at short stimulation periods [15]. In a paced cable or tissue alternans appears in two major forms, concordant (CA) and discordant (DA) alternans, depending on if the phase of the alternation is the same along the tissue or it presents nodes (in 1D) or nodal lines (in 2D) separating regions of tissue oscillating out of phase. For normal conduction, at constant pacing period, concordant alternans are transformed into discordant alternans if the tissue size is increased [16]. A good understanding of this transition is particularly relevant since discordant alternans is widely acknowledged as a precursor to potentially life-threatening arrythmias like tachycardia and fibrillation $[17,18]$.

In the present work we study two examples of dynamics in a cardiac model that are strongly affected by SNC. First, we consider propagation of two and more pulses on a ring and show that SNC destabilizes periodic excitation patterns and leads to pulse pairs and pulse bunching. Second, we demonstrate that concordant alternans (CA) is promoted and is observed in simulations of arbitrarily long paced one-dimensional cables. This phenomenon is theoretically explained by an amplitude equation approach [19]. A second important consequence is that SNC allows for period doubled spirals with straight defect lines (in contrast to spiraling defect lines in cardiac models with normal conduction [20]). Hence, the experimental observation of straight defect lines in period-doubled spirals in cell cultures of cardiac rat cells [21] can be explained by the presence of SNC.

We consider the cable equation for propagation of the action potential:

$$
\partial_{t} V_{m}=D \nabla^{2} V_{m}-I_{\text {ion }} / C_{m}
$$

with $V_{m}$ the transmembrane potential, $I_{\text {ion }}$ given by the DRBR model [10], the diffusion constant $D=0.972 \times 10^{-3} \mathrm{~cm}^{2} / \mathrm{ms}$ [11], and the membrane capacitance $C_{m}=1 \mu \mathrm{F} / \mathrm{cm}^{2}$. We perform numerical simulations of Eq. (1) [22] in a $1 \mathrm{D}$ ring and paced cable as well as in 2D to study the effect of SNC on pulse trains and spirals. For the ring we also use a continuation 


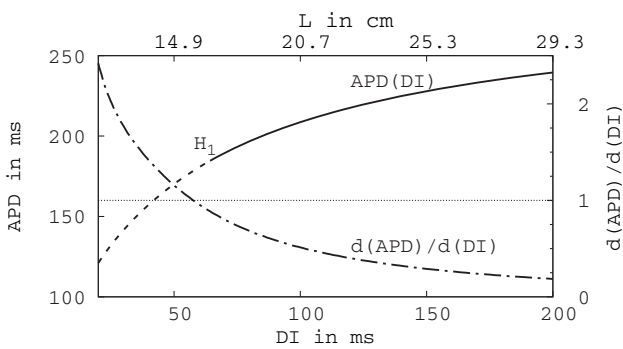

FIG. 1. Action potential duration (APD) restitution curve and derivative of the APD with respect to DI plotted against the diastolic interval and wavelength $\mathrm{L}$, for a steadily moving single pulse on a ring. The APD and DI are measured from the steepest increase of the transmembrane potential to a repolarization of $90 \%$. Solid (dashed) APD denotes stable (unstable) periodic pulse trains.

method [11] that gives access to the stability of traveling waves by a linear stability analysis. In Fig. 1 we show the action potential duration (APD) restitution curve found this way for a single pulse in the ring. The corresponding dispersion relation is shown in Fig. 2(a). The single pulse is stable for ring length above the critical value at the Hopf instability $H_{1}$ to alternans, which occurs in the region of negative slope of the dispersion curve. Beyond the Hopf, the solution is always in the form of DA with a node evolving slowly in time, similar to what is observed for normal CV restitution [23].

A more interesting situation develops when two pulses are allowed in the ring. In this case the negative slope of the dispersion curve results in an attractive interaction between the pulses. This gives rise to a new branch of asymmetric pulse pairs [Fig. 2(b)] starting at a period-doubling bifurcation $P D$. The periodic pulse solution is never stable and there is a direct transition from the branch of bound (period-doubled) states to modulated waves (DA) at the Hopf instability point $\mathrm{H}_{2}$. The resulting states are shown in Fig. 3. It must be stressed that this type of period-doubled waves appear because of the positive interpulse attraction due to the SNC and not due to the alternans instability. The distinction is made clearer if we consider several pulses in the ring (Fig. 4). Then the
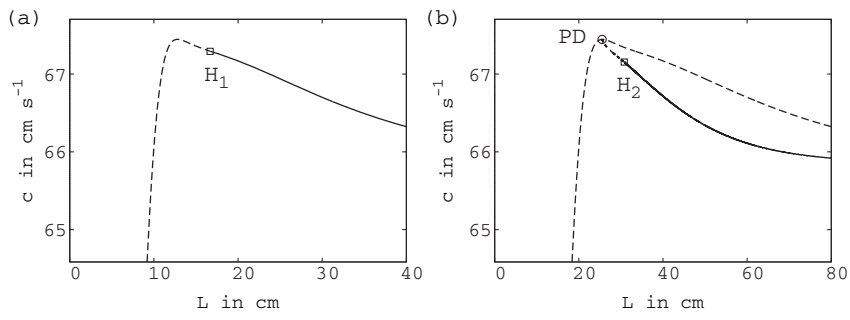

FIG. 2. Dispersion relation and bifurcation diagram (conduction velocity vs ring perimeter) of (a) a single pulse and (b) a pair of pulses on a ring. The solid (dashed) lines indicate stable (unstable) solutions. Decreasing the length of the ring, the single pulse becomes unstable at a Hopf bifurcation $H_{1}$ that appears in the region with negative slope $c^{\prime}(L)<0$. In (b) the dashed line describes an unstable pulse pair consisting of two identical pulses at a distance $L / 2$. A branch of asymmetric pulse pairs bifurcates from a period doubling bifurcation $P D$. Increasing $L$, it gains its stability at a Hopf bifurcation $\mathrm{H}_{2}$ at a ring length $L_{H_{2}}>L_{P D}$.
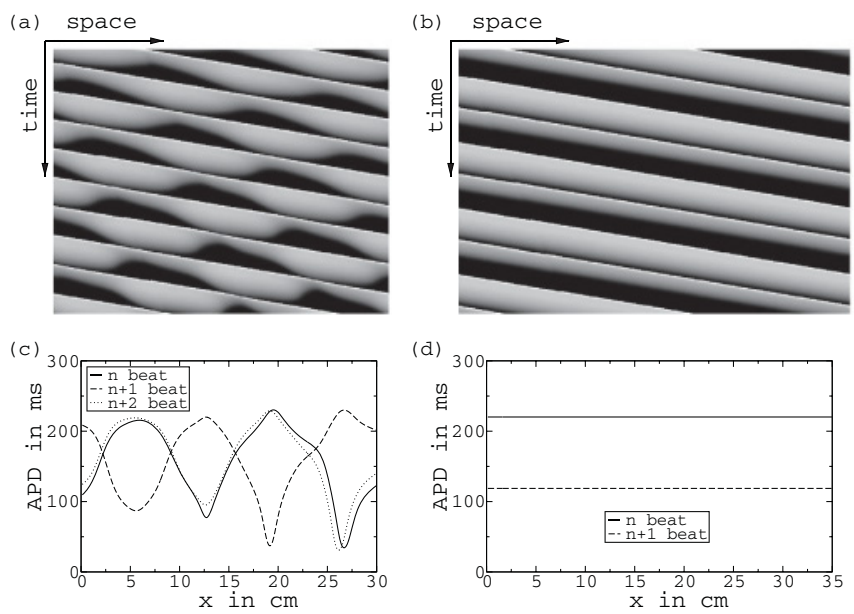

FIG. 3. Space-time plot of the voltage for a pair of pulses on a ring with (a) $L=30 \mathrm{~cm}<L_{H_{2}}$ and (b) $L=35 \mathrm{~cm}>L_{H_{2}}$. In the lower panels the spatial distributions of APD as a function of distance along the cable corresponding to (c) DA and (d) CA are shown.

simulations exhibit pulse bunching, that is, all action potentials form a bound state [6].

In a paced cable the periodic pulse trains are found to be stable for large values of the pacing period. Decreasing the period, the first bifurcation results in CA (see Fig. 5). This is in contrast to the case of normal conduction where alternans gives rise to DA for sufficiently long cables. In this latter case, alternans effectively appear as a result of a (near or strictly resonant) 2:1 Hopf bifurcation with a finite wave number such that CA can only be observed if the length of the system is shorter than a fourth of the wavelength of the resulting pattern [19]. For SNC, CA is always the most unstable mode, independently of the length of the system. However, a transition to DA is observed decreasing the pacing period [Figs. 5(c) and 5(d)], eventually resulting in 2:1 block for small periods [Fig. 5(e)].

The appearance of concordant alternans in the case of SNC can be understood within the amplitude equation formalism derived in [19]. The dynamics of the $n$th AP close to the onset of cardiac alternans is given by $\mathrm{APD}^{n} \simeq \mathrm{APD}_{c}+a(x) e^{i \pi n}$, where $\mathrm{APD}_{c}$ is the action potential duration right at the onset of alternans. The amplitude $a(x)$ of the slow modulations of the period doubling is described by the equation

$$
T \partial_{t} a=\sigma a-w \partial_{x} a+\xi^{2} \partial_{x}^{2} a-g a^{3}-\kappa \int_{0}^{x} a\left(x^{\prime}\right) d x^{\prime},
$$

where $T$ is the period of pacing and $\sigma$ is proportional to the distance to onset. The quantities $w$ and $\xi$ are characteristic
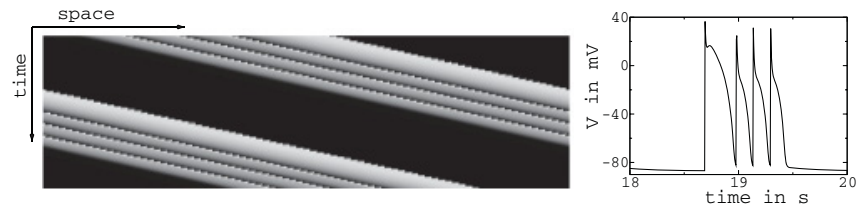

FIG. 4. Numerical simulations of Eq. (1) in a long cable $(L=$ $100 \mathrm{~cm}$ ) showing four pulses that form a stable bunch of pulses with a practically constant profile (right) that propagates at constant speed (see space-time plot on left). 

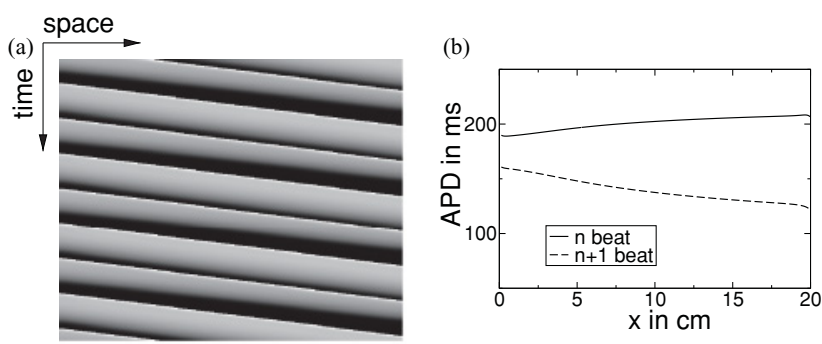

(c) space

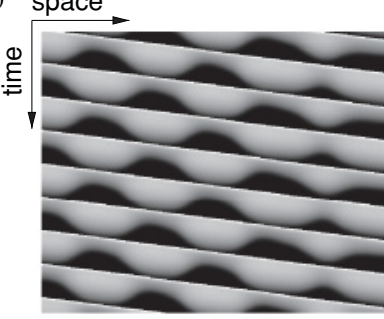

(d)

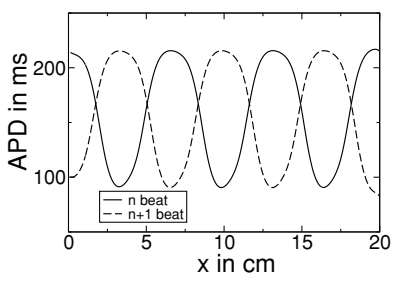

(e)

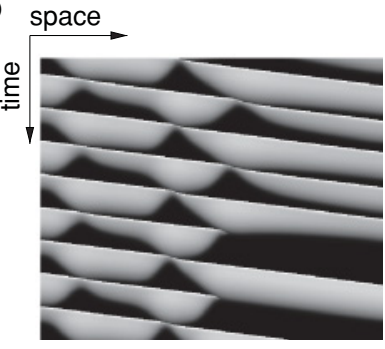

(f)

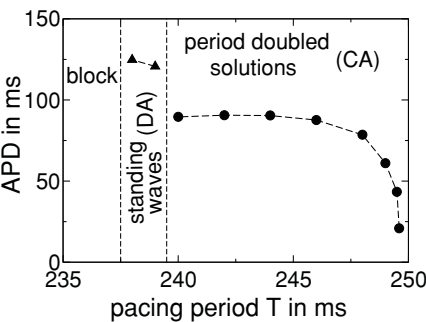

FIG. 5. Simulations in a cable of length $L=20 \mathrm{~cm}$ with a periodic stimulation $T$ at $x=0$. Decreasing the pacing period $T$, a period-doubled (CA) solution appears [shown in (a) and (b) at $T=249 \mathrm{~ms}]$ up to a period of $T \simeq 239 \mathrm{~ms}$, where it is replaced by discordant alternans (DA) [(c) and (d) at $T=238 \mathrm{~ms}$. For still shorter periods, conduction block appears first far from the pacing point [(e) at $T=236 \mathrm{~ms}$ ] then resulting into $2: 1$ block. In (f) we show the amplitude of oscillation as a function of pacing period $T$.
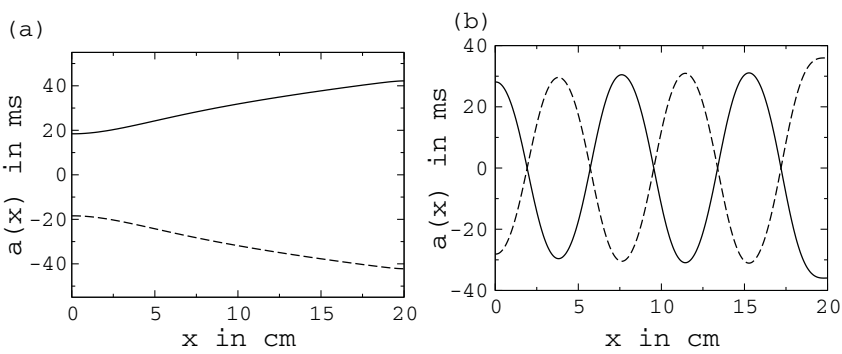

FIG. 6. Amplitude of alternans as a function of space obtained with simulations of the amplitude equation (2) for (a) negative and (b) positive dispersion. Solid and dashed lines $[a(x)$ and $-a(x)$, respectively] would correspond to the amplitude at two consecutive beats for established solutions once transients have died out, showing (a) CA and (b) DA in the form of standing waves. The parameters are $\xi=0.2 \mathrm{~cm}^{2}, w=0.03 \mathrm{~cm}, g=1.78 \times 10^{-5} \mathrm{~ms}^{-2}$, and (a) $\sigma=0.004, \kappa=-0.002 \mathrm{~cm}^{-1}$, (b) $\sigma=0.04, \kappa=0.02 \mathrm{~cm}^{-1}$. These parameters are chosen so as to obtain similar amplitude and wavelength of alternans solutions as in the full numerical simulations.
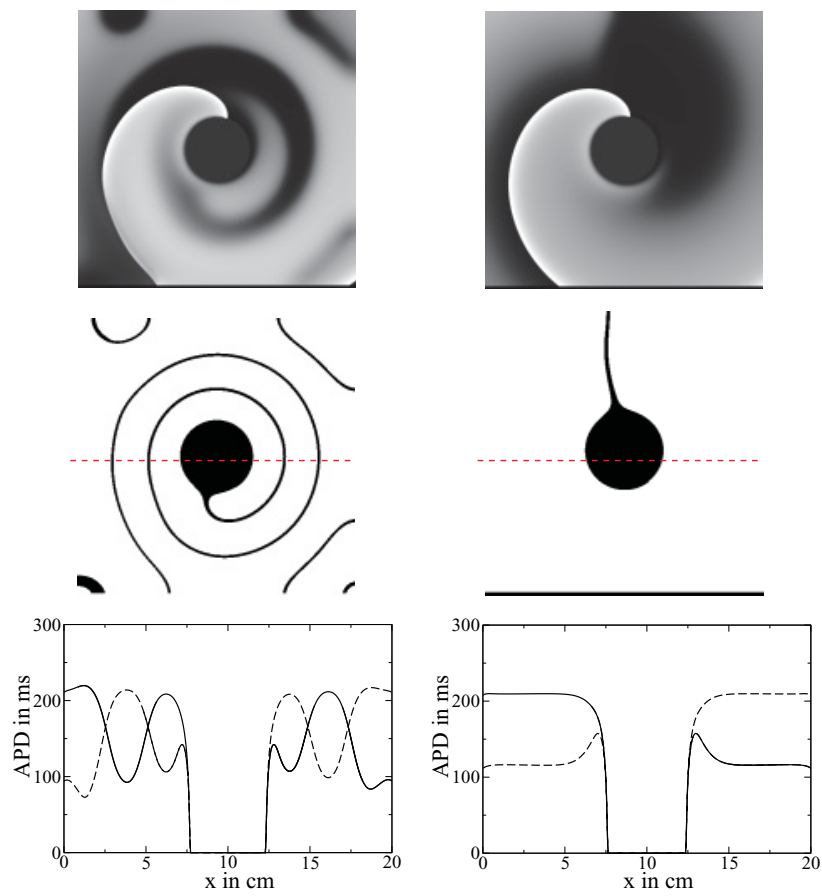

FIG. 7. Pinned spirals with an unexcitable circle of radius $r=$ $2.28 \mathrm{~cm}$ (left panels) and $r=2.36 \mathrm{~cm}$ (right panels), producing periods of the spirals of $T \simeq 237 \mathrm{~ms}$ and $T=244 \mathrm{~ms}$. At the unexcitable circle the value of the transmembrane voltage is kept fixed at $V_{m}=-80 \mathrm{mV}$. In the middle panels we only plot the points on which the difference between two consecutive APDs is smaller than $18 \mathrm{~ms}$. We show a snapshot of the transmembrane voltage, the nodal line(s), and the APD along the dashed red line in the middle panels.

length scales associated with diffusion, and $\kappa=2(d c / d \mathrm{DI}) / c^{2}$ is related to the slope of $\mathrm{CV}$ restitution. The case of normal dispersion $(\kappa>0)$ was studied in [19], showing that the nonlocal term in Eq. (2) nucleates defects, giving rise to Turing-like patterns with a well-defined wavelength. For SNC $(\kappa<0)$ on the contrary, its effect is to promote the stabilization of CA. Changing $\kappa$ from negative (SNC) to positive (normal conduction) $\mathrm{CA}$ is replaced by DA (Fig. 6).

For spiral waves, the transition from DA to CA drastically changes the form and dynamics of nodal lines (defined as those points whose APD does not change from beat to beat). Topological reasons imply that spirals undergoing an alternans transition always exhibit a nodal line (or an odd number of them) emerging from the core. These nodal lines can have complex dynamics including nucleation and splitting [24]. While the dynamics and form of such nodal lines or synchronization defect lines are relatively well understood for complexoscillatory media where a 2:1 resonant Hopf bifurcation yields similar phenomena [25] as alternans here, the situation is less clear for excitable media [20,21,26,27]. In cultures of neonatal rat myocytes, for instance, both static and rotating nodal lines have been observed [21]. The dynamics of the nodal line can be affected by meander, medium inhomogeneities, or conduction velocity restitution. Since our main focus is on this last effect, we have performed simulations of pinned 
spiral waves in which we change the size of a nonexcitable core to change the properties of the spiral. In Fig. 7 we show simulations of pinned spirals at different rotation periods. As in the cable, in the radial direction a transition is observed from CA which in this case results in straight defect lines to DA. The latter ones correspond to spiraling defect lines that rotate slowly.

In summary, we have studied alternans in the DRBR ionic model by means of simulations and bifurcation analysis. This model displays alternans due to steep APD restitution for wave trains in the regime of SNC. In that regime, pulses on a ring exhibit an attractive interaction which results in asymmetric pulse pairs and pulse bunching in simulations. Numerical bifurcation analysis shows the destabilization of symmetric pulse pairs and periodic pulse trains due to the attractive interaction as well as the emergence of an asymmetric pulse pair from a period doubling bifurcation near the maximum of the CV restitution curve. Simulations of a periodically paced cable show that concordant alternans appears as the pacing period is decreased. For higher pacing frequencies modulated waves (DA) appear. A related transition appears in the radial dynamics of spiral waves undergoing a transition to alternans, whose nodal line changes from being straight to spiraling as the frequency of the spiral is increased. Theoretical analysis by means of an amplitude equation shows that these phenomena are not confined to the specific model used here but are generically expected for all cardiac models that exhibit SNC near the onset of alternans. Our results are highly relevant to excitable media and to all experiments where SNC is found in cardiac tissue (see, e.g., Ref. [13]). With respect to alternans, the experimental observation of straight defect lines in period-doubled spirals in cell cultures of cardiac rat cells [21] can be explained mechanistically by the presence of SNC.

B.E. wants to acknowledge financial support by MICINN (Spain) under project Grant No. FIS2008-06335-C02-01. M.B. and H.E. acknowledge support by DFG via SFB-CRC 555 and GRK-RTG 1558. M.B. and B.E. also thank ESF Funcdyn, DAAD, and MICINN for travel support. J.D. was supported by NERSC. B.E. and J.D. thank PTB for hospitality.
[1] J. J. Tyson and J. P. Keener, Physica D 32, 327 (1988); E. Meron, Phys. Rep. 218, 1 (1992).

[2] E. M. Cherry and F. H. Fenton, Am. J. Physiol. Heart Circ. Physiol. 286, 2332 (2004).

[3] J. F. Spear and E. N. Moore, Circ. Res. 35, 782 (1974).

[4] J. Christoph et al., Phys. Rev. Lett. 82, 1586 (1999).

[5] C. T. Hamik, N. Manz, and O. Steinbock, J. Phys. Chem. A 105, 6144 (2001).

[6] M. Or-Guil, I. G. Kevrekidis, and M. Bär, Physica D 1350, 154 (2000).

[7] C. T. Hamik and O. Steinbock, New J. Phys. 5, 1 (2003); G. Bordyugov, N. Fischer, H. Engel, N. Manz, and O. Steinbock, Physica D 239, 766 (2010).

[8] F. H. Fenton, E. M. Cherry, H. M. Hastings, and S. J. Evans, Chaos 12, 852 (2002).

[9] V. Iyer, R. Mazhari, and R. L. Winslow, Biophys. J. 87, 1507 (2004); A. Bueno-Orovio, E. M. Cherry, and F. H. Fenton, J. Theor. Biol. 253, 544 (2008).

[10] G. W. Beeler and H. Reuter, J. Physiol. (London) 268, 177 (1977); J. P. Drouhard and F. A. Roberge, Comp. Biomed. Res. 20, 333 (1987).

[11] S. Bauer, G. Röder, and M. Bär, Chaos 17, 015104 (2007).

[12] C. H. Luo and Y. Rudy, Circ. Res. 68, 1501 (1991); E. de Lange and J. P. Kucera, Biophys J. 96, 294 (2009).

[13] E. de Lange and J. P. Kucera, Biophys. J. 98, 1129 (2010).

[14] O. Kwon, T. Y. Kim, and K. J. Lee, Phys. Rev. E 82, 046213 (2010).
[15] T. Lewis, Q. J. Med. 4, 141 (1910).

[16] M. A. Watanabe, F. H. Fenton, S. J. Evans, H. M. Hastings, and A. Karma, J. Cardiovasc. Electrophys. 12, 196 (2001).

[17] J. M. Pastore, S. D. Girouard, K. R. Laurita, F. G. Akar, and D. S. Rosenbaum, Circulation 99, 1385 (1999).

[18] J. N. Weiss et al., Circulation 112, 1232 (2005).

[19] B. Echebarria and A. Karma, Phys. Rev. Lett. 88, 208101 (2002); Phys. Rev. E 76, 051911 (2007).

[20] J. G. Restrepo and A. Karma, Phys. Rev. E 79, 030906 (2009).

[21] T. Y. Kim, S. J. Woo, S. M. Hwang, J. H. Hong, and K. J. Lee, Proc. Natl. Acad. Sci. USA. 104, 11639 (2007).

[22] We discretize Eq. (1) using a simple forward explicit Euler method with $d x=0.01 \mathrm{~cm}$ and $d t=0.02 \mathrm{~ms}$.

[23] M. Courtemanche, L. Glass, and J. P. Keener, Phys. Rev. Lett. 70, 2182 (1993); M. Courtemanche, J. P. Keener, and L. Glass, SIAM J. Appl. Math. 56, 119 (1996).

[24] J.-S. Park and K. J. Lee, Phys. Rev. Lett. 83, 5393 (1999); J.-S. Park, S. J. Woo, O. Kwon, T. Y. Kim, and K. J. Lee, ibid. 100, 068302 (2008).

[25] A. Goryachev, R. Kapral, and H. Chaté, Int. J. Bif. Chaos 10, 1537 (2000); J. Davidsen, R. Erichsen, R. Kapral, and H. Chate, Phys. Rev. Lett. 93, 018305 (2004); B. Sandstede and A. Scheel, SIAM J. Appl. Dyn. Syst. 6, 494 (2007).

[26] M. Yoneyama, A. Fujii, and S. Maeda, J. Am. Chem. Soc. 117, 8188 (1995).

[27] A. Goryachev and R. Kapral, Int. J. Bif. Chaos 9, 2243 (1999). 